EOMmun Communication et organisation

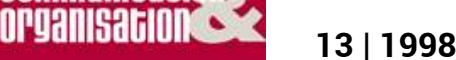

Management par projet et logiques

communicationnelles

\title{
Management par projet : communiquer et après ?
}

François Jolivet

\section{OpenEdition}

Journals

Édition électronique

URL : http://journals.openedition.org/communicationorganisation/2050

DOI : 10.4000/communicationorganisation.2050

ISSN : $1775-3546$

Éditeur

Presses universitaires de Bordeaux

Édition imprimée

Date de publication : 1 mai 1998

ISSN : 1168-5549

Référence électronique

François Jolivet, « Management par projet : communiquer et après ? ", Communication et organisation

[En ligne], 13 | 1998, mis en ligne le 26 mars 2012, consulté le 05 mai 2019. URL : http://

journals.openedition.org/communicationorganisation/2050 ; DOI : 10.4000/

communicationorganisation. 2050

Ce document a été généré automatiquement le 5 mai 2019.

(c) Presses universitaires de Bordeaux 


\title{
Management par projet : communiquer et après?
}

\author{
François Jolivet
}

\section{Communiquer c'est respirer}

1 Première réaction en recevant l'invitation à «faire un papier » sur la communication dans les projets : ce sujet me paraît être une tarte à la crème relevant de la logique du style « tout homme armé d'un marteau considère que tout problème est un clou ».

2 Communiquer c'est comme respirer, on voit pas comment s'en passer. Puis me reviennent en mémoire quelques situations vécues.

\section{Celui qui gagne de l'argent a raison, celui qui en perd a tort}

3 1967. J'ai 28 ans, je viens d'être nommé patron d'un chantier d'autoroute dans la plaine de Saône. Venant du bureau d'études, j'avais été détaché sur le chantier pour six mois : je devais faire les plans d'étaiement des 18 ponts. Mais voilà, six mois après, le patron du chantier «se fait virer ", ça marchait mal et sa relation avec le directeur basé à Lyon auquel il venait d'être rattaché, était orageuse. Difficulté à communiquer?

4 Je me suis depuis cette date forgé une logique primaire : « quand on gagne de l'argent, on a raison, quand on en perd on a tort ». La qualité de la communication dépend de la santé économique du projet ou de celle de l'entreprise. Ceci m'a fait comprendre pourquoi les livres de management vieillissent mal : ils font appel à des « success stories ».

5 Mais revenons sur l'autoroute. 


\section{Dialoguer avant de décider.}

6 Je fus nommé directeur des travaux : j'étais le patron de 600 personnes, je n'avais jamais exercé auparavant de commandement. Problème numéro un, la solitude du chef. Je m'en suis sorti en dialoguant avec un de «mes » collaborateurs, qui est devenu un ami. Fils d'un émigré italien, il avait commencé comme tailleur de pierres, puis avait gravi tous les échelons pour devenir conducteur de travaux : il était en charge de la construction des 18 ponts (la section d'autoroute comportait outre les ponts, trois millions de mètres cubes de terrassements à déplacer). Il m'apprit toutes les ficelles du métier et me révéla la formidable capacité à modifier le cours des événements, capacité que chacun a en soi.

7 Il m'a fallu dissocier la discussion de la décision: la discussion est collective, un seul prend la décision. Je n'ai toujours pas compris comment on arrivait à prendre de bonnes décisions en recherchant le consensus. Sony a fait en France la même constatation: le consensus à la française c'est un "consensus mou » qui conduit à des décisions médiocres. Donc un seul doit décider : ce qui implique d'avoir le courage d'aller contre l'avis de ses collaborateurs, mais en connaissance de cause. Pour que le dialogue soit fructueux, il ne faut-il pas se laisser manipuler (faire des recoupements), ou prendre plaisir à avoir des collaborateurs « cireurs de pompes ». Mon opinion sur le sujet : on a les collaborateurs qu'on mérite.

Deuxième enseignement de cette époque : ne pas avoir honte de revenir sur une décision quand elle s'avère mauvaise. C'est difficile la première fois de dire à ses collaborateurs : « je me suis planté, on arrête ». Quand on l'a fait une fois ce n'est plus un problème ; on garde cependant suffisamment d'amour propre pour éviter de se planter trop fréquemment. Et puis, si on veut que les collaborateurs vous parlent des problèmes, de leurs erreurs, il faut bien donner l'exemple. Ce qui fait dire à un P.-D.G. très branché sur l'exemplarité: «les collaborateurs copient leur chef, dommage qu'ils copient plus les défauts que les qualités ».

Troisième enseignement: les chefs ne doivent pas «jouer au chef». Il faut gommer la distance hiérarchique, tout en restant responsable de ses propres actes. Ce n'est pas facile car, comme dans un pièce de théâtre, chacun dans l'entreprise joue un rôle. Rester lucide implique une pointe d'humour envers soi.

\section{On a l'opinion de son fauteuil.}

L'utilisation de métaphores et de l'humour sont essentiels pour bien communiquer. Illustrons par exemple la phrase « on a l'opinion de son fauteuil », phrase qui explique bien des comportements.

11 En voyant les pyramides d'Egypte, le chef d'entreprise pensera : «cela a été une réussite, parce qu'à la tête de tout cela, il y avait un pharaon». Le directeur de projet pensera : " voilà un beau projet, certes pas rentable dans l'immédiat, mais à long terme, le retour sur investissement se fait par le tourisme ». Quant au financier sorti de l'ENA, il pensera : « la forme même des pyramides laisse présumer une restriction budgétaire ». Enfin le directeur des ressources humaines pensera tout bas : «dans cette affaire, l'essentiel était la gestion des carrières!»" 
12 L'humour permet de garder un éclair de lucidité et de bien communiquer. De même, raconter une histoire vécue, un épisode plus ou moins embelli, une «histoire d'ancien combattant » est un moyen de communiquer (partager) avec son équipe.

\section{3 minutes, 3 heures, 3 jours.}

Mais revenons à la prise de décision. Comment structurer la communication avant de prendre la décision? Le succès de la conduite de projet repose sur la responsabilisation et la projection de l'activité (préparation du travail, simulation). Venant du bureau d'études, je m'étais fixé comme règle de faire le travail 3 fois : en 3 minutes, en 3 heures et en 3 jours. En 3 minutes, c'est se poser les questions suivantes: Quel est le problème? Quelles sont les solutions? En 3 heures, c'est projeter la solution avec des méthodes rapides (ratios, esquisse, croquis). En 3 jours, c'est réaliser l'ouvrage (dans le cas présent une étude).

La communication associée à une prise de décisions peut être structurée de la même façon (si elle n'est pas structurée, cela tourne très vite à une discussion de «café du commerce »). Première étape: le questionnement verbal. Cela oblige à projeter mentalement l'activité et à rechercher les solutions. Deuxième étape: la projection visualisée par croquis, chiffrage, programme, maquette. Cette démarche oblige l'autre à être précis, à découvrir les difficultés. Troisième étape : la réalisation, le physique. La vue de l'ouvrage fait découvrir un autre éclairage, et permet de capitaliser avec les deux premières étapes : la boucle de feed-back génère la compétence future : "Comment ça s'est passé ? Est-ce conforme à ce que nous avions projeté ?». La veille de visiter un chantier réalisé par un confrère, je me pose les questions : comment je ferais? quelle solution adopter? Une fois, je ne m'étais pas posé de question : je n'ai rien vu lors de la visite !

\section{Les dix premières années}

15 J'ai acquis la conviction que les dix premières années de la vie professionnelle déterminent tout le reste. Ce n'est que plus tard que j'ai compris pourquoi. Lors des dix premières années professionnelles, on apprend à identifier les problèmes, à communiquer, à projeter l'activité, à affronter l'adversité ; on prend plaisir à transformer sa pensée en actions et en résultats. Un scientifique dirait que les premières années forgent les processus cognitifs (le mot est à la mode) : les processus d'apprentissage, de prise de décisions et de communication.

\section{Stopper la communication est quelquefois utile}

16 Quelques années plus tard, après le chantier de l'autoroute, j'ai apprécié les vertus de la non-communication. Rattaché au directeur basé à Lyon (précédemment cité), je devais construire un pont et un parc de stationnement en pleine ville. Le directeur régional insista pour que je m'installe dans ses bureaux (situé à 800 mètres du chantier). Je considérais que ma place était au milieu de «mon » équipe et de «mon" chantier, mais vu la proximité de « ses » bureaux, je n'avais pas le choix. 
17 Tous les matins, je retrouvais sur mon bureau des notes manuscrites de mon patron me demandant des explications, critiquant certaines décisions, bref me harcelant. J'ai découvert à cette occasion que, lorsqu'on grimpe dans la hiérarchie on continue à faire le boulot de la fonction antérieure : il avait été un excellent patron de chantier, il voulait faire mon travail ou me montrer qu'il savait (bien) le faire. Je décidais de couper la communication : il eut la surprise en rentrant d'un voyage au siège parisien de trouver mon bureau vide, débarrassé de tout mobilier et de la secrétaire (qui était également l'agent administratif du chantier). J'étais allé m'installer en bord de Saône, dans une baraque de chantier. C'était l'épreuve de force. Pendant un an, nous ne nous sommes plus parlé ; je lui envoyais les informations relevant de sa fonction sous forme de notes ; il regardait le chantier depuis le pont voisin; je m'efforçais de ne pas mettre en évidence cette crise devant mes collaborateurs, c'était peine perdue, chacun était au courant et observait. J'étais certain que si le chantier passait dans le rouge, « il » ne me " louperait » pas. Je n'avais pas d'autre issue que le succès : quelle merveilleuse motivation !

Tout cela n'est pas nouveau: Jules César avait brûlé ses vaisseaux à Alexandrie pour n'avoir comme seule issue que la victoire ; quant à Blériot, il ne savait pas nager, cela a dû le motiver à réussir la traversée de la Manche. J'ai remarqué depuis que beaucoup de responsables et de patrons se mettaient consciemment ou inconsciemment dans ce genre de situation.

\section{Comment ne pas faire le travail de ses collaborateurs?}

19 Autre réflexion datant de cette époque : « comment éviter de faire le travail de la fonction précédente » ? Certains ont trouvé la solution ou plutôt des solutions :

- commencer à faire chaque matin la partie du travail que l'on n'aime pas,

21 - avoir 25 à 30 collaborateurs en direct,

22 - avoir une fonction mixte : dans un groupe de 500 filiales, le directeur régional, qui doit superviser cinq à six entreprises, est en même temps le patron de l'une d'elles. Il est « à la fois capitaine et lieutenant ». Efficace : non seulement, il n'enm... plus ses collaborateurs, mais il se voit dans le miroir!

23 - s'éloigner de ses collaborateurs. C'est ce qu'à fait le P.-D.G.d'un groupe de 35000 personnes. Conscient de sa pulsion naturelle à mettre la pression sur ses patrons de branche d'activité, il a décidé de s'installer dans un lieu géographique distinct et éloigné.

24 Quel rapport avec la communication? Si vous n'empiétez pas sur la responsabilité de vos collaborateurs, le dialogue sera meilleur. C.Q.F.D.! Dialoguer ne doit pas entraîner un transfert de responsabilités.

\section{La communication au quotidien dans un projet}

Mais revenons à la communication dans les projets. En 75, je suis affecté au siège parisien d'une entreprise pour m'occuper des offres concernant la construction des centrales nucléaires en Iran et en Afrique du Sud. 


\section{la communication à l'intérieur
partenaires du groupement?}

On me désigne un bureau déjà occupé ; je découvre après quelques jours que mon vis-àvis est en charge de la même mission que moi. La proximité aidant, le boulot ne manquant pas, nous décidâmes de nous partager le travail plutôt que de nous affronter.

27 J'ai appris plus tard que l'ambiguïté de mon nouveau patron était volontaire : il voulait me tester, il voulait voir comment j'allais réagir et me débrouiller. Faut-il manier l'ambiguïté avec ses collaborateurs? Avec le recul, je n'en suis toujours pas partisan; il est vrai que si on est trop précis, le collaborateur attend tout de son chef : il vaut mieux qu'il se focalise plus sur le projet que sur son chef.

Un an plus tard, nous décrochons le contrat des centrales nucléaires d'Afrique du Sud, en groupement d'entreprises avec Framatome et Alsthom. Je suis nommé responsable de la part du contrat revenant à mon entreprise (Spie-Batignolles).

La fonction de chef de projet n'existait pas dans l'activité génie-civil (essentiellement dévolue à la construction). Il était d'usage de désigner au siège un « ingénieur en charge » pour apporter le soutien nécessaire au chantier. Il s'agissait pour ce contrat d'une fonction de chef de projet, puisque la responsabilité contractuelle était globale: conception/construction, clés en mains, prix forfaitaire, responsabilité de performance conjointe et solidaire avec les deux autres partenaires du groupement. Sur place, en Afrique du Sud, est nommé un directeur des travaux, sans que soit tranché la question de savoir : qui est rattaché à qui ? Toujours l'ambiguïté !

Le projet doit se développer sur deux fronts : d'une part l'ingénierie, les relations entre partenaires et avec le client, d'autre part la construction proprement dite. Je constitue à Paris une équipe d'environ trente personnes, je mobilise trois bureaux d'études, l'équipe doit piloter l'ingénierie, les bureaux d'études, préparer, négocier et superviser les approvisionnements de matériaux et les activités de fabrication réalisée en France.

Comment gérer tout cela, sans référentiel, sans expérience d'ingénierie ? Comment gérer 作 Nous ne nous sommes pas posé de questions à ce sujet: le projet a la vertu de tirer l'action, c'est une véritable force motrice. Cela m'a conduit quelques années plus tard à définir le management par projet comme étant la capacité à subordonner l'organisation, les processus, les comportements aux finalités du projet. Je réalise maintenant que j'aurais pu rajouter la communication.

33 Bref, nous nous sommes progressivement auto-organisés : j'avais appris en chantier l'efficacité du découpage des responsabilités par ouvrage (la décomposition du projet en éléments physiques).

Je rassemblais l'équipe des trente personnes dans un même lieu géographique. Ce n'est pas un problème mineur, chacun connait les difficultés pour obtenir la libération de l'espace! Quand on libère une place dans un siège, il faut en général bouger trois, voire quatre personnes - c'est ce que nous appelions le jeu du taquin - ce petit jeu où une seule case est libre.

35 Comment organiser l'équipe? Chaque mois, je mettais à jour une page représentant l'organigramme : c'était la liste alphabétique de l'équipe des trente personnes (mon nom commençant par J, j'étais au milieu), la mission de chacun étant définie en quelques mots (pas de phrase). Il y avait cinq responsables de sous-projets, les autres personnes servant de support de compétence: études générales, achats, contrats, secrétariat, 
documentation, expertise technique, qualité, etc. Pas de réunions structurées, toutes les portes ouvertes. Premier enseignement : pour que les gens collaborent, il faut qu'ils aient un objectif, qu'ils sachent qui est responsable de quoi, qui doit prendre telle décision. Il faut également que le décideur soit accessible. Il faut que le projet prime sur la notion hiérarchique : chacun a sa propre responsabilité. Le centrage sur le projet impose de gommer toute la symbolique hiérarchique : la dimension des bureaux, la moquette, les titres et les comportements féodaux : le chef voit ses collaborateurs dans leurs bureaux, le café est fait pour tout le monde, etc.

Mais alors, quid la communication? Elle n'était pas organisée. Par contre tout le monde savait (de façon plus ou moins précise) où en était le projet. Tout le monde pouvait communiquer avec tout le monde: dans cette équipe de trente personnes, les réunions étaient toujours improvisées, peu nombreuses, sans compte rendu, par contre les communications externes étaient structurées.

\section{Organiser, regrouper pour pouvoir communiquer}

Un constat : quand chacun sait ce qu'il a à faire, la communication peut s'établir. Si les rôles sont mal définis, si les objectifs ne sont pas clairs, alors il y a toutes les raisons pour que cela se bloque. Attention, définir le rôle de chacun, n'est pas écrire une «job description » à l'américaine de deux pages : la finalité n'est pas le territoire de chacun, c'est le projet : chacun doit apprendre à se positionner, sans que cela génère des luttes stériles. Il faut apprendre à faire le travail de l'autre quand il est en congé ou surchargé : la solidarité naît de la participation à un but commun.

Bien sûr, tout ne se déroula pas à la perfection. En particulier, deux des chefs de sousprojets ne s'entendaient pas. Il venait me voir séparément pour me demander de prendre position sur leurs interfaces communs. Je n'entendais pas tomber dans le piège : chacun devait coordonner son activité avec les autres. Sans dire pourquoi, j'organisai un changement de bureaux (il y avait en général deux ingénieurs par bureau) afin que les deux chefs de sous-projets se retrouvent au quotidien, l'un en face de l'autre. Ce fut radical. J'ai usé (abusé ?) de cette méthode plusieurs fois.

Lors d'un autre conflit, je décidais d'envoyer les deux protagonistes ensemble en mission: rien de tel que 48 heures passées en déplacement pour se découvrir mutuellement (en voyage, on sort du jeu de rôle).

\section{Sandwiches et boîtes de bière}

Quant à la communication dans le groupement, les matières à difficultés ne manquaient pas : le contrat et la convention de groupement n'étaient pas précis, il y avait des sommes en jeu importantes, les risques contractuels étaient élevés (pénalités de performance, de retard, travaux additionnels induits par un partenaire), nous ne savions pas exactement ce que nous avions vendu et par dessus tout les relations entre les P.-D.G. des trois sociétés n'étaient pas au beau fixe (c'est le moins qu'on puisse dire). Bref, un projet avec trois chefs de projets, trois logiques d'entreprise qui s'affrontent, mais un seul client, un seul projet.

41 Le petit jeu des groupements consiste à maximiser les retombées pour sa société et à transférer les difficultés chez ses partenaires, ce qui entraîne des comportements 
déviants, alors que la résolution des problèmes nécessite un effort de chacun simultané ou à tour de rôle. 1 à 10 pour un même projet. J'ai découvert les vertus du découpage du projet en sous- 
projets au lieu et place du découpage fonctionnel (études, achats, fabrication, qualité, coûts, délais etc.).

Dans le découpage en sous-projets, un chef de sous-projet est responsable de l'intégration de tous les aspects fonctionnels du sous-projets, il va prendre les centaines, voire les milliers de micro-décisions concernant le sous-projet.

Cette coordination se fait dans sa tête, au lieu de se faire dans des dizaines, voire des centaines de réunions. La coordination entre sous-projets se fait par les objectifs. Les interfaces entre sous-projets se règlent entre deux personnes physiques. Pour peu que l'on découpe intelligemment les sous-projets, en laissant à l'intérieur la complexité, on réduit considérablement la communication.

Cela me rappelle un souvenir d'ingénieur débutant au bureau d'études. J'étais allé voir l'ingénieur en chef en lui disant: «j'ai un problème et je ne sais pas le résoudre ». Il me répondit : « supprimer le problème ». Génial. Si vous avez des problèmes de complexité de communication, supprimez les!

Que dire d'autre à propos de la communication? L'écrit oblige à être plus précis, tout le monde le sait, mais ne s'y oblige pas. Mettre ses idées sur le papier oblige à les clarifier.

\section{Reformuler}

Il faudrait aussi un mécanisme de feed-back pour vérifier que l'autre a bien compris. Je n'ai pas trouvé de bonne formule. Cela paraît désobligeant de demander à son interlocuteur de reformuler ce qu'on vient de lui dire. Si on reformule soi-même, on a du mal à trouver une autre présentation. Je suis pourtant arrivé à la conclusion que s'obliger à reformuler de façon différente, est un excellent exercice. Difficile, néanmoins.

\section{Le contrat « pousse - au - crime »}

La spirale de dégradation des relations entre client et fournisseur est un vrai problème : si elle est enclenchée, il est difficile d'en sortir. Cette spirale peut exister entre individus, ou entre sociétés. Les contrats "poussent au crime ", surtout depuis le développement du juridique dans l'entreprise, sous l'influence des pratiques anglo-saxonnes. Le projet implique une notion de partenariat, cela impose que chacun soit professionnel, joue son rôle, dans l'adversité, comme au rugby, mais aussi que chacun puisse dialoguer après la sortie des vestiaires ou lors de la troisième mi-temps. Il faut un esprit constructif et que chacun gagne de l'argent !

On voit de plus en plus d'entreprises, de maîtres d'ouvrage et de maîtres d'œuvre nourrir durant toute la vie du projet un dossier contentieux. C'est basé sur la conviction qu'un contrat est un affrontement entre deux intérêts contradictoires, qui ne peut se terminer que par un conflit juridique : d'où des comportements suicidaires. On préfère renvoyer le problème chez l'autre plutôt que de s'asseoir autour de la table pour le résoudre : c'est ainsi qu'un début d'incendie qui pouvait s'éteindre avec un verre d'eau devient un énorme brasier. Cela s'appelle gérer le contrat plutôt que gérer le projet. Le problème est amplifié par les contrats «one shot » où le fournisseur et le client n'ont pas de relations continues. Quand les relations sont basées sur la méfiance, le soupçon, les accusations, 
c'est mal parti (d'autant plus que certains conseilleurs, maitre-d'œuvre ou autre, peuvent avoir intérêt à dramatiser la situation pour valoriser leur rôle !

Tout ceci génère une spirale de dégradation, où les conflits prennent de plus en plus d'ampleur et où, in fine, tout le monde est perdant. Le tunnel sous la Manche a été très marqué par ce genre de situation (entre tous les acteurs principaux). Il faudra attendre une dizaine d'années, voire plus, pour pouvoir en tirer à froid des enseignements.

La spirale de dégradation des relations fait penser aux études menées par le Mental Research Center de Palo Alto (Californie), études qui traitent de la communication entre individus (et non entre sociétés). J'ai tenté dans certaines circonstances d'appliquer les remèdes préconisés : recadrage, comportement paradoxal. Sans succès. Voilà un bon sujet pour les chercheurs.

Le matriciel : un parfait générateur de conflits stériles

61 Je ne parlerai pas des jeux de pouvoirs dans l'entreprise, des luttes de territoires, des batailles rangées et sournoises entre les métiers et les projets. Quel gâchis! L'organisation matricielle est un magnifique générateur de conflits: vous pouvez organiser la communication, elle ne fonctionnera pas tant que vous n'aurez pas traité la cause première : l'ambiguïté de la définition des responsabilités. Il faut répondre aux questions : qui décide ? Qui est responsable ? Celui qui est responsable a-t-il la maitrise des moyens ? Je continue à penser que les dirigeants passent une large partie de leur temps à régler des problèmes qu'ils ont eux-mêmes créés !

\section{Communiquer via la gastronomie}

63 Je n'ai pas parlé des moyens et outils qui favorisent la communication. J'en citerai deux : la proximité et le fax. Ils sont toujours d'actualité, malgré le nouvel engouement pour le multimédia et les réseaux informatiques. Parmi les autres moyens, je citerai la fête (savoir faire la fête après l'effort, c'est consolider des relations qui vont permettre de traiter les problèmes futurs).

Dans un registre similaire, le P.-D.G. cité plus haut prend plaisir à répéter : «je pratique la communication via la gastronomie ».

\section{Marcel Duchamp}

Qu'il me soit permis d'évoquer Marcel Duchamp, jugé par certains, comme l'artiste le plus important du vingtième siècle. "Ce sont les regardeurs qui font les tableaux » a-t-il dit, pour souligner que l'on ne sait pas dissocier la chose observée de l'observateur. Mon énumération de quelques souvenirs d'«ancien combattant», illustre, plus modestement, que la communication ne peut être séparée de son contexte et du sens que chacun donne aux propos et à l'attitude d'autrui. 


\section{BIBLIOGRAPHIE}

Du même auteur :

F. JOLIVET, «The possibility of anticipating, several years in advance, the success or failure of a project » in : Project Management Institute symposium, Montréal, Québec, Canada, September 1986. pp. 35/39.

F. JOLIVET - C. Navarre, « Grands projets, auto-organisation, méta-règles : vers de nouvelles

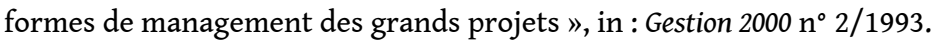

F. JOLIVET, « L'entreprise » orientée projet ». L'apprentissage des organisations » in : Gestion 2000 n ○ 6/1995.

F. JOLIVET, « Peut-on éviter les dysfonctionnements? », in : L'Expansion Management Review, Mars

95

F. JOLIVET, «L'organisation matricielle en question. Nouvelles relations métiers/projet », in : Cahiers IQM 1998.

F. JOLIVET, « Management de projet. Et si on parlait vrai ? », publication prévue en 98.

\section{RÉSUMÉS}

L'auteur décrit, à travers des situations particulières qu'il a vécues, les problèmes de communication rencontrés. Les constats effectués sont la qualité de la communication est fonction de la santé économique du projet et de celle de l'entreprise, la qualité d'une décision dépend du dialogue qui la précède et de la capacité à ne pas rechercher le consensus, les situations de blocage peuvent pousser à se surpasser, la clarification des responsabilités est un préalable à une bonne communication, le découpage des responsabilités par sous-projets évite d'avoir à gérer une communication complexe, l'esprit positif est indispensable pour gérer une communication client/fournisseur. La communication est indissociable du contexte et du sens donné par chacun des communicants.

The author describes the various communication problem encountered in specific situations he has experienced. His observations are as follows : the quality of communication depends of the economies health of the project, and the company as a whole, the quality of a decision depends of a discussion preceding it and a capacity for not trying to reach a consensus at all costs, situations which seem to be bloc-ked may push people to outdo themselves, responsibilities need to be clarified to achieve good communication. Communication can be simplified by dividing responsibilities into sub-projects, a positive attitude is vital in managing customer/supplier relations. Communication is also dependent on the context and the meaning given by the various people involved. 


\section{AUTEUR}

\section{FRANÇOIS JOLIVET}

L'auteur est un praticien de la conduite de projet dans le BTP. Parmi les projets dont il a eu la responsabilité, figurent la construction de plusieurs sections d'autoroute en France, de bâtiments dans différents pays en Afrique, Amérique du Sud, Moyen et Extrême Orient, de centrales nucléaires en Afrique du Sud, du tunnel sous la Manche. L'auteur a engagé une réflexion et des recherches sur la pertinence et l'efficacité des méthodes de management de projet, ce qui a conduit au concept d'auto-organisation autour de métarègles. 